\title{
QUASI-NEUTRAL LIMIT OF EULER-POISSON SYSTEM OF COMPRESSIBLE FLUIDS COUPLED TO A MAGNETIC FIELD
}

\author{
JIANWEI YANG
}

\begin{abstract}
In this paper, we consider the quasi-neutral limit of a three dimensional Euler-Poisson system of compressible fluids coupled to a magnetic field. We prove that, as Debye length tends to zero, periodic initial-value problems of the model have unique smooth solutions existing in the time interval where the ideal incompressible magnetohydrodynamic equations has smooth solution. Meanwhile, it is proved that smooth solutions converge to solutions of incompressible magnetohydrodynamic equations with a sharp convergence rate in the process of quasi-neutral limit.
\end{abstract}

\section{INTRODUCTION}

The main objective of this paper is to study the quasi-neutral limit of the following Euler-Poisson system of compressible fluids coupled to a magnetic field [6, 21]:

$$
\begin{aligned}
& \partial_{t} n^{\lambda}+\operatorname{div}\left(n^{\lambda} u^{\lambda}\right)=0 \text { in } \mathbb{T}^{3}, t>0, \\
& \partial_{t}\left(n^{\lambda} u^{\lambda}\right)+\operatorname{div}\left(n^{\lambda} u^{\lambda} \otimes u^{\lambda}\right)+\nabla p\left(n^{\lambda}\right)=n^{\lambda} \nabla \phi^{\lambda}+\operatorname{curl} B^{\lambda} \times B^{\lambda}, \\
& \partial_{t} B^{\lambda}-\operatorname{curl}\left(u^{\lambda} \times B^{\lambda}\right)=0, \\
& \operatorname{div} B^{\lambda}=0, \\
& \lambda^{2} \Delta \phi^{\lambda}=n^{\lambda}-1,
\end{aligned}
$$

with initial conditions:

$$
n^{\lambda}(\cdot, 0)=n_{0}^{\lambda}, u^{\lambda}(\cdot, 0)=u_{0}^{\lambda}, B^{\lambda}(\cdot, 0)=B_{0}^{\lambda} .
$$

In the above equations, $\mathbb{T}^{3}$ is 3 -dimensional torus and $\lambda>0$ is the (scaled) Debye length. The unknown functions are the density $n^{\lambda}$, the velocity $u^{\lambda}=\left(u_{1}^{\lambda}, u_{2}^{\lambda}, u_{3}^{\lambda}\right)$, the magnetic field $B^{\lambda}=\left(B_{1}^{\lambda}, B_{2}^{\lambda}, B_{3}^{\lambda}\right)$ and the gravitational potential $\phi^{\lambda}$. Throughout this paper, we assume that the pressure function $p\left(n^{\lambda}\right)$ satisfies the usual $\gamma$-law,

$$
p\left(n^{\lambda}\right)=\frac{\left(n^{\lambda}\right)^{\gamma}}{\gamma}, n^{\lambda}>0
$$

for some constant $\gamma>1$. It is obvious that equations(1.4) is redundant with equations (1.3), as soon as they are satisfied by the initial conditions $\operatorname{div} B_{0}^{\lambda}=0$. System (1.1)-(1.5) is used to model the evolution of a magnetic stars 3 . The effects

Date: July 11, 2018.

2010 Mathematics Subject Classification. 35B25, 35Q35, 35Q40.

Key words and phrases. Euler-Poisson system, magnetic field, Quasi-neutral limit, Incompressible magnetohydrodynamic equations, Energy estimate . 
of magnetic fields arise in some physically interesting and important phenomena in astrophysics; e.g. solar flares. Without taking magnetic effects into account, system (1.1)-(1.5) reduces to the Euler-Poisson equations.

In recent years, the quasi-neutral limit $(\lambda \rightarrow 0)$ of various models has attracted much attention. In particular, the limit $\lambda \rightarrow 0$ has been performed in VlasovPoisson system by Brenier [1], Grenier [11,13] and Masmoudi [19], in drift-diffusion equations by Gasser et al. [7, 8 and Jüngel and Peng [9], and in the one dimensional and isothermal Euler-Poisson system by Cordier and Grenier [2], in more general isentropic models by Wang [23, in non-isentropic Euler-Poisson equations by Peng et al. [20] and Li [15], in Euler-Monge-Ampère systems by Loeper [17, in NavierStokes-Poisson system by Wang and Jiang [24, Donatelli and Marcati [5] and Ju et al. 10, in quantum hydrodynamics equations [14, in Navier-Stokes-Fourier-Poisson system by Li et al. [16, etc. As far as we know, there is no result on quasi-neutral limit of the Euler-Poisson system coupled to a magnetic field (1.1)-(1.5).

In this paper, we will study the quasi-neutral limit for the smooth solution of the system (1.1)-1.5) in the framework of the convergence-stability principle developed in 26. Formally, taking the (scaled) Debye length $\lambda \rightarrow 0$ in (1.5), we obtain the following ideal incompressible magnetohydrodynamic equations

$$
\begin{aligned}
& \partial_{t} u^{0}+\left(u^{0} \cdot \nabla\right) u^{0}+\nabla p^{0}=\operatorname{curl} B^{0} \times B^{0}, \\
& \partial_{t} B^{0}-\operatorname{curl}\left(u^{0} \times B^{0}\right)=0, \\
& \operatorname{div} u^{0}=\operatorname{div} B^{0}=0 .
\end{aligned}
$$

The objective of this paper is to make this limit rigorous. Our proof requires the (local) existence of a smooth solution to (1.1)-(1.5), which is shown in next section. The proof of our result is based on the convergence-stability principle developed by Yong [25, 26] for singular limit problems of symmetrizable hyperbolic systems. In contrast with the results in $1,2,5,10,14,17,23,24$, where the limiting equations are incompressible Euler equations or the incompressible Navier-Stokes equations, our limiting equations are the incompressible magnetohydrodynamic equations (1.7)(1.9). In our case where the Euler-Poisson equations are coupled to a magnetic field, the problem becomes more challenging. Because of the magnetic field and non-linearity terms, some elaborated energy analysis are required to obtain the desired convergence results.

This paper is organized as follows. In section 2, we rewrite the system (1.1)-(1.5) as a symmetrizable hyperbolic system to obtain the local-in-time existence result, and present our main results. The proof of Theorem 2.4 is obtained in section 3.

Before ending the introduction, we give the notation and Lemma used throughout the current paper. The letters $C$ and $C_{T}$ denote various positive constants independent of $\lambda$, which can be different from one line to another one, but $C_{T}$ may depend on $T$. The symbol ":" means summation over both matrix indices. $|U|$ denotes some norm of a vector or matrix $U$. Also, we denote

$$
\|\cdot\|=\|\cdot\|_{L^{2}\left(\mathbb{T}^{3}\right)},\|\cdot\|_{\infty}=\|\cdot\|_{L^{\infty}\left(\mathbb{T}^{3}\right)},\|\cdot\|_{k}=\|\cdot\|_{H^{k}\left(\mathbb{T}^{3}\right)}, k \in \mathbb{N}^{*} .
$$


QUASI-NEUTRAL LIMIT OF EULER-POISSON SYSTEM COUPLED TO A MAGNETIC FIELD

Lemma 1.1. (see, e.g. [18]). Let $s, s_{1}$, and $s_{2}$ be three nonnegative integers and $s_{0}=\left[\frac{d}{2}\right]+1$.

1. If $f, g \in H^{s} \cap L^{\infty}$ and any nonnegative multi index $\beta,|\beta| \leq s$, then we have

$$
\left\|D^{\beta}(f g)\right\| \leq C_{s}\left(\|f\|_{L^{\infty}}\left\|D^{\beta} g\right\|+\|g\|_{L^{\infty}}\left\|D^{\beta} f\right\|\right) \leq c_{s}\|f\|_{s}\|g\|_{s} .
$$

2. If $f \in H^{s}$, $D f \in L^{\infty}, g \in H^{s-1} \cap L^{\infty}$, then we have

$$
\left\|D^{\beta}(f g)-f D^{\beta} g\right\| \leq C_{s}\left(\|D f\|_{L^{\infty}}\left\|D_{x}^{\beta^{\prime}} g\right\|+\|g\|_{L^{\infty}}\left\|D^{\beta} f\right\|\right),\left|\beta^{\prime}\right|=|\beta|-1 \text {. }
$$

3. Let $s_{3}=\min \left\{s_{1}, s_{2}, s_{1}+s_{2}-s_{0}\right\} \geq 0$, then $H^{s_{1}} H^{s_{2}} \subset H^{s_{3}}$. Here the inclusion symbol $\subset$ implies the continuity of the embedding.

4. Suppose $s \geq s_{0}, A \in C_{b}^{s}(G)$, and $U \in H^{s}(\Omega, G)$. Then $A(U(\cdot)) \in H^{s}$ and

$$
\|A(U(\cdot))\|_{s} \leq C_{s}|A|_{s}\left(1+\|U\|_{s}^{s}\right) .
$$

Here and below, $C_{s}$ denotes a generic constant depending only on $s$ and $d$, and $|A|_{s}$ stands for $\sup _{\{U \in G,|\alpha| \leq s\}}\left|\partial_{U}^{\alpha} A(U)\right|$.

\section{Main Results}

First, we consider the local existence of smooth solution of the system (1.1)-(1.5) for any fixed $\lambda>0$.

By Green's formulation, it follows from (1.1) and (1.5) that

$$
\nabla \phi^{\lambda}=\frac{1}{\lambda^{2}}\left(\nabla \Delta^{-1}\left(n_{0}^{\lambda}-1\right)-\nabla \Delta^{-1} \operatorname{div} \int_{0}^{t}\left(n^{\lambda} u^{\lambda}\right)(x, \tau) d \tau\right) .
$$

Using (1.1) and the following equality

$$
\operatorname{div}\left(n^{\lambda} u^{\lambda} \otimes u^{\lambda}\right)=n^{\lambda}\left(u^{\lambda} \cdot \nabla\right) u^{\lambda}+u^{\lambda} \operatorname{div}\left(n^{\lambda} u^{\lambda}\right),
$$

we can rewrite (1.2) as

$$
\partial_{t} u^{\lambda}+\left(u^{\lambda} \cdot \nabla\right) u^{\lambda}+\nabla h\left(n^{\lambda}\right)=\nabla \phi^{\lambda}+\frac{1}{n^{\lambda}} \operatorname{curl} B^{\lambda} \times B^{\lambda},
$$

where the enthalpy $h\left(n^{\lambda}\right)>0$ is defined by

$$
h^{\prime}\left(n^{\lambda}\right)=\frac{p^{\prime}\left(n^{\lambda}\right)}{n^{\lambda}}
$$

for $n^{\lambda}>0$.

Set

$$
\begin{gathered}
W^{\lambda}=\left(\begin{array}{c}
n^{\lambda} \\
u^{\lambda} \\
B^{\lambda}
\end{array}\right), W_{0}^{\lambda}=\left(\begin{array}{c}
n_{0}^{\lambda} \\
u_{0}^{\lambda} \\
B_{0}^{\lambda}
\end{array}\right), \\
A_{i}\left(W^{\lambda}\right)=\left(\begin{array}{ccc}
u_{i}^{\lambda} & n^{\lambda} e_{i}^{T} & 0 \\
h^{\prime}\left(n^{\lambda}\right) e_{i} & u_{i}^{\lambda} \mathbf{I}_{3 \times 3} & \frac{\left(G_{i}^{\lambda}\right)^{T}}{n^{\lambda}} \\
0 & \frac{G_{i}^{\lambda}}{n^{\lambda}} & u_{i}^{\lambda} \mathbf{I}_{3 \times 3}
\end{array}\right), \\
F^{\lambda}=\left(\begin{array}{c}
\nabla \Delta^{-1}\left(n_{0}^{\lambda}-1\right)-\nabla \Delta^{-1} \operatorname{div} \int_{0}^{t}\left(n^{\lambda} u^{\lambda}\right)(x, \tau) d \tau \\
0
\end{array}\right),
\end{gathered}
$$


where $\left(e_{1}, e_{2}, e_{3}\right)$ is the canonical base of $\mathbb{R}^{3}, \mathbf{I}_{3 \times 3}$ is a unit matrix, $y_{i}$ denotes the $i$ th component of $y \in \mathbb{R}^{3}$ and

$$
\begin{gathered}
G_{1}^{\lambda}=\left(\begin{array}{ccc}
0 & 0 & 0 \\
B_{2}^{\lambda} & -B_{1}^{\lambda} & 0 \\
B_{3}^{\lambda} & 0 & -B_{1}^{\lambda}
\end{array}\right), G_{2}^{\lambda}=\left(\begin{array}{ccc}
-B_{2}^{\lambda} & B_{1}^{\lambda} & 0 \\
0 & 0 & 0 \\
0 & B_{3}^{\lambda} & -B_{2}^{\lambda}
\end{array}\right), \\
G_{3}^{\lambda}=\left(\begin{array}{ccc}
-B_{3}^{\lambda} & 0 & B_{1}^{\lambda} \\
0 & -B_{3}^{\lambda} & B_{2}^{\lambda} \\
0 & 0 & 0
\end{array}\right) .
\end{gathered}
$$

Thus the problem (1.1)-(1.6) for the unknown $W$ can be rewritten as

$$
\begin{aligned}
& \partial_{t} W^{\lambda}+\sum_{i=1}^{3} A_{i}\left(W^{\lambda}\right) \partial_{x_{i}} W^{\lambda}=\frac{1}{\lambda^{2}} F^{\lambda}, \\
& \left.W^{\lambda}\right|_{t=0}=W_{0}^{\lambda} .
\end{aligned}
$$

It is not difficult to see that the equations of $W^{\lambda}$ in (2.3)-(2.4) are symmetrizable hyperbolic, i.e. if we introduce

$$
A_{0}\left(n^{\lambda}\right)=\left(\begin{array}{ccc}
h^{\prime}\left(n^{\lambda}\right) & 0 & 0 \\
0 & n^{\lambda} \mathbf{I}_{3 \times 3} & 0 \\
0 & 0 & n^{\lambda} \mathbf{I}_{3 \times 3}
\end{array}\right),
$$

which is positively definite when $n^{\lambda}>\delta>0$, then $\widehat{A}_{i}\left(W^{\lambda}\right)=A_{0}\left(n^{\lambda}\right) A_{i}\left(W^{\lambda}\right)$ are symmetric for all $1 \leq i \leq 3$.

Thus, to solve the system (1.1)-(1.6), it suffices to solve the system (2.3)-(2.4). Since the non-local source term $\nabla \Delta^{-1} \operatorname{div} \int_{0}^{t}\left(n^{\lambda} u^{\lambda}\right)(x, \tau) d \tau$ is a sum of products of Riesz transforms of $\int_{0}^{t}\left(n^{\lambda} u^{\lambda}\right)(x, \tau) d \tau$, we have, by the $L^{2}$ boundedness of the Riesz transformation (see [22]),

$$
\left\|\nabla \Delta^{-1} \operatorname{div} \int_{0}^{t}\left(n^{\lambda} u^{\lambda}\right)(x, \tau) d \tau\right\|_{s} \leq C\left\|\int_{0}^{t}\left(n^{\lambda} u^{\lambda}\right)(x, \mu) d \mu\right\|_{s},
$$

for some constant $C>0$ independent of $t$.

Moreover, we recall the other elementary fact which can be easily proven by using Fourier series.

Lemma 2.1. $\nabla \Delta^{-1}$ is a bounded linear operator from $V=\left\{v \in L^{2}\left(\mathbb{T}^{3}\right) \mid \boldsymbol{m}(v)=0\right\}$ into $H^{1}\left(\mathbb{T}^{3}\right)$.

So one gets

$$
\left\|\nabla \Delta^{-1}\left(n_{0}^{\lambda}-1\right)\right\|_{s+1} \leq C\left\|\left(n_{0}^{\lambda}-1\right)\right\|_{s}
$$

for some constant $C>0$. Based on the above crucial facts, using the standard iteration techniques of local existence theory for symmetrizable hyperbolic systems (see [18), we have

Proposition 2.2. Assume that the initial conditions $\left(n_{0}^{\lambda}, u_{0}^{\lambda}, B_{0}^{\lambda}\right) \in H^{s}\left(\mathbb{T}^{3}\right), s>$ $\frac{5}{2}, n_{0}>\delta>0, \operatorname{div} B_{0}^{\lambda}=0$ and $\int_{\mathbb{T}}^{3}\left(n_{0}^{\lambda}-1\right) d x=0$. Then for any fixed $\lambda>0$, there exists a positive constant $T$ (may depend on $\lambda$ ) such that the periodic problem 
(2.3)-(2.4) has a unique smooth solution $\left(n^{\lambda}, u^{\lambda}, B^{\lambda}\right) \in C\left([0, T] ; H^{s}\left(\mathbb{T}^{3}\right)\right)$, welldefined on $\mathbb{T}^{3} \times[0, T]$. Hence the nonlinear periodic problem (1.1)-(1.6) admits a unique solution $\left(n^{\lambda}, u^{\lambda}, \nabla \phi^{\lambda}, B^{\lambda}\right)$ satisfying

$$
\left(n^{\lambda}, u^{\lambda}, \nabla \phi^{\lambda}, B^{\lambda}\right) \in C\left([0, T] ; H^{s}\left(\mathbb{T}^{3}\right)\right) .
$$

According to Proposition 2.2, for each fixed $\lambda>0$ in (1.1)-(1.6), there exists a time interval $[0, T]$ such that system (1.1)-(1.6) has a unique solution $\left(n^{\lambda}, u^{\lambda}, \nabla \phi^{\lambda}, B^{\lambda}\right)$ satisfying (2.5). Define

$$
\begin{aligned}
& T_{\lambda}=\sup \left\{T>0:\left(n^{\lambda}, u^{\lambda}, \nabla \phi^{\lambda}, B^{\lambda}\right) \in C\left([0, T] ; H^{s}\left(\mathbb{T}^{3}\right)\right),\right. \\
& \left.\frac{1}{2} \leq n^{\lambda} \leq \frac{3}{2}, \forall(x, t) \in \mathbb{T}^{3} \times[0, T]\right\} .
\end{aligned}
$$

Namely, $\left[0, T_{\lambda}\right]$ is the maximal time interval of $H^{s}$ - existence. Note that $T_{\lambda}$ may tend to zero as $\lambda$ goes to 0 . In order to show that $\liminf _{\lambda \rightarrow 0} T_{\lambda}>0$, we follow the convergence-stability principe [26] and seek a formal approximation of $\left(n^{\lambda}, u^{\lambda}, B^{\lambda}\right)$. To this end, we consider the initial-value problem of the ideal incompressible magnetohydrodynamic equation (1.7)-(1.9) with initial data

$$
u^{0}(\cdot, 0)=u_{0}^{0}, B^{0}(\cdot, 0)=B_{0}^{0}
$$

Let us recall the local existence of a strong solution to the ideal incompressible magnetohydrodynamic equations (1.7)-(1.9). The proof can be found in [4].

Proposition 2.3. (see [4].) Let $s>\frac{5}{2}$ be an integer. Assume that the initial data

$$
\left.\left(u^{0}(x, t), B^{0}(x, t)\right)\right|_{t=0}=\left(u_{0}^{0}(x), B_{0}^{0}(x)\right)
$$

satisfy

$$
\left(u_{0}^{0}, B_{0}^{0}\right) \in H^{s+1}\left(\mathbb{T}^{3}\right) \text { and } \operatorname{div} u_{0}^{0}=0, \operatorname{div} B_{0}^{0}=0 .
$$

Then, there exist a $T_{*} \in(0,+\infty)$ and a unique solution $\left(u^{0}, B^{0}\right) \in L^{\infty}\left(\left[0, T_{*}\right), H^{s+1}\left(\mathbb{T}^{3}\right)\right)$ to the ideal incompressible magnetohydrodynamic equations (1.7)-(1.9) satisfying, for any $0<T<T_{*}$,

$$
\operatorname{div} u^{0}=0, \operatorname{div} B^{0}=0
$$

and

$$
\sup _{0 \leq t \leq T}\left(\left\|\left(u^{0}, B^{0}\right)\right\|_{s+1}+\left\|\left(\partial_{t} u^{0}, \partial_{t} B^{0}\right)\right\|_{s}+\left\|\nabla p^{0}\right\|_{s+1}+\left\|\partial_{t} \nabla p^{0}\right\|_{s}\right) \leq C_{T}
$$

for some positive constant $C_{T}$.

Now the main result of this paper reads as follows.

Theorem 2.4. Let $s>\frac{3}{2}+1$ be an integer. Suppose $\operatorname{div} u_{0}^{0}=0, \operatorname{div} B_{0}^{0}=0$, and incompressible magnetohydrodynamic equations (1.7)-(1.9) with the initial data $\left(u_{0}^{0}, B_{0}^{0}\right)$ has a solution $\left(u^{0}, B^{0}\right) \in L^{\infty}\left(\left[0, T_{*}\right), H^{s+1}\left(\mathbb{T}^{3}\right)\right)$. Then, for $\lambda$ sufficiently small, there exists a $\lambda$-independent positive number $T_{* *}<T *$, such that the model (1.1) -(1.5) with periodic initial data $\left(n_{0}^{\lambda}, u_{0}^{\lambda}, B_{0}^{\lambda}\right)$ satisfying

$$
n_{0}^{\lambda}=1, u_{0}^{\lambda}=u_{0}^{0}, B_{0}^{\lambda}=B_{0}^{0},
$$


has a unique solution $\left(n^{\lambda}, u^{\lambda}, B^{\lambda}, \nabla \phi^{\lambda}\right) \in C\left(\left[0, T_{* *}\right] ; H^{s}\left(\mathbb{T}^{3}\right)\right)$. Moreover, there exists a $\lambda$-independent constant $M>0$ such that

$$
\sup _{0 \leq t \leq T}\left(\left\|n^{\lambda}-1\right\|_{s}+\left\|u^{\lambda}-u^{0}\right\|_{s}+\left\|B^{\lambda}-B^{0}\right\|_{s}\right) \leq M \lambda .
$$

Remark 2.1. The initial data

$$
n_{0}^{\lambda}=1, u_{0}^{\lambda}=u_{0}^{0}, B_{0}^{\lambda}=B_{0}^{0}
$$

can be relaxed as

$$
n_{0}^{\lambda}=1+O\left(\lambda^{2}\right), u_{0}^{\lambda}=u_{0}^{0}+O(\lambda), B_{0}^{\lambda}=B_{0}^{0}+O(\lambda),
$$

without changing our arguments.

Remark 2.2. Theorem 2.4 describes the quasi-neutral limit $\lambda \rightarrow 0$ of the system (1.1)-(1.5) with well-prepared initial data, avoiding the presence of the initial time layer. We will discuss the case of general initial data (ill-prepared initial data) allowing the presence of the fast singular oscillation in the future.

\section{Proof of Theorem 2.4}

Thanks to the convergence-stability principle developed in [25,26], it suffices to prove the error estimate in (2.10) for $t \in\left[0, \min \left\{T_{\lambda}, T_{* *}\right\}\right]$ with $T_{* *}<T_{*}$ independent of $\lambda$ and to be determined. Thus we directly make the error estimate (2.10) in the time interval $\left[0, \min \left\{T_{\lambda}, T_{* *}\right\}\right]$.

Now we rewrite (2.3) as the following form

$$
\begin{aligned}
& \partial_{t} W^{\lambda}+\sum_{i=1}^{3} A_{i}\left(W^{\lambda}\right) \partial_{x_{i}} W^{\lambda}=\left(\begin{array}{c}
0 \\
\nabla \phi^{\lambda} \\
0
\end{array}\right), \\
& \lambda^{2} \Delta \phi^{\lambda}=n^{\lambda}-1, \operatorname{div} B^{\lambda}=0 .
\end{aligned}
$$

We note that with $\left(u^{0}, p^{0}, B^{0}\right)$ constructed in Proposition 2.3.

$$
\left(n_{\lambda}, u_{\lambda}, \phi_{\lambda}, B_{\lambda}\right)=\left(1, u^{0},-p^{0}, B^{0}\right)
$$

satisfies

$$
\begin{aligned}
& \partial_{t} n_{\lambda}+\operatorname{div}\left(n_{\lambda} u_{\lambda}\right)=0, \\
& \partial_{t} u^{\lambda}+\left(u_{\lambda} \cdot \nabla\right) u_{\lambda}+\nabla h^{\prime}\left(n_{\lambda}\right)=\nabla \phi_{\lambda}+\frac{1}{n_{\lambda}} \operatorname{curl} B_{\lambda} \times B_{\lambda}, \\
& \partial_{t} B_{\lambda}-\operatorname{curl}\left(u_{\lambda} \times B_{\lambda}\right)=0 \\
& \operatorname{div} B_{\lambda}=0 \\
& \lambda^{2} \Delta \phi_{\lambda}=n_{\lambda}-1-\lambda^{2} \Delta Q
\end{aligned}
$$

with $Q=-p^{0}$. From Proposition 2.3 we have

$$
\sup _{t \in\left[0, T_{*}\right]}\left(\|\nabla Q(\cdot, t)\|_{s+1}+\left\|\partial_{t} \nabla Q(\cdot, t)\right\|_{s}\right)<+\infty .
$$


So, we can rewrite (3.3)-(3.7) as

$$
\begin{gathered}
\partial_{t} W_{\lambda}+\sum_{i=1}^{3} A_{i}\left(W_{\lambda}\right) \partial_{x_{i}} W_{\lambda}=\left(\begin{array}{c}
0 \\
\nabla \phi_{\lambda} \\
0
\end{array}\right), \\
\lambda^{2} \Delta \phi_{\lambda}=n_{\lambda}-1-\lambda^{2} \Delta Q, \operatorname{div} B^{\lambda}=0 .
\end{gathered}
$$

Set

$$
E=W^{\lambda}-W_{\lambda}=\left(\begin{array}{c}
N \\
U \\
H
\end{array}\right)=\left(\begin{array}{c}
n^{\lambda}-n_{\lambda} \\
u^{\lambda}-u_{\lambda} \\
B^{\lambda}-B_{\lambda}
\end{array}\right), \Phi=\lambda\left(\phi^{\lambda}-\phi_{\lambda}\right) .
$$

We deduce from (3.1)-(3.2) and (3.8)-(3.9) that

$$
\begin{aligned}
& \partial_{t} E+\sum_{i=1}^{3} A_{i}\left(W^{\lambda}\right) \partial_{x_{i}} E=\frac{1}{\lambda} G+\sum_{i=1}^{3}\left(A_{i}\left(W^{\lambda}\right)-A_{i}\left(W_{\lambda}\right)\right) \partial_{x_{i}} W_{\lambda}, \\
& \lambda \Delta \Phi=N+\lambda^{2} \Delta Q,
\end{aligned}
$$

where

$$
G=\left(\begin{array}{c}
0 \\
\nabla \Phi \\
0
\end{array}\right)
$$

We differentiate (3.10) with $\partial_{x}^{\alpha}$ for a multi-index $\alpha$ satisfying $|\alpha| \leq s$ with $s>\frac{5}{2}$ to get

$$
\partial_{t} E_{\alpha}+\sum_{i=1}^{3} A_{i}\left(W^{\lambda}\right) \partial_{x_{i}} E_{\alpha}=\frac{1}{\lambda} G_{\alpha}+R_{\alpha}^{1}+R_{\alpha}^{2}
$$

with $\partial_{x}^{\alpha} f=f_{\alpha}$, where

$$
\begin{gathered}
R_{\alpha}^{1}=\sum_{i=1}^{3}\left[\left(A_{i}\left(W^{\lambda}\right)-A_{i}\left(W_{\lambda}\right)\right) \partial_{x_{i}} W_{\lambda}\right]_{\alpha} \\
R_{\alpha}^{2}=\sum_{i=1}^{3}\left[A_{i}\left(W^{\lambda}\right) \partial_{x_{i}} E_{\alpha}-\left(A_{i}\left(W^{\lambda}\right) \partial_{x_{i}} E\right)_{\alpha}\right] .
\end{gathered}
$$

For the sake of clarity, we divide the following arguments into lemmas.

Lemma 3.1. Under the conditions of Theorem 2.4, we have

$$
\begin{aligned}
& \frac{d}{d t} \int_{\mathbb{T}^{3}}\left(E_{\alpha}^{T} A_{0}\left(n^{\lambda}\right) E_{\alpha}+\left|\nabla \Phi_{\alpha}\right|^{2}\right) d x \\
& \leq C\|\nabla \Phi\|_{s}^{2}+\frac{C}{\lambda}\|U\|_{s}\|N\|_{s}\left\|\nabla \Phi_{\alpha}\right\|+\frac{2}{\lambda}\|E\|_{s}\left\|U_{\alpha}\right\|\left\|\nabla \Phi_{\alpha}\right\| \\
& \quad+C\left\|E_{\alpha}\right\|\left\|R_{\alpha}^{1}\right\|+C\left\|E_{\alpha}\right\|\left\|R_{\alpha}^{2}\right\|+C\left(1+\|E\|_{s}\right)\left\|E_{\alpha}\right\|^{2}+\lambda^{2},
\end{aligned}
$$

where $C$ is a generic constant depending only on the range $\left(\frac{1}{2}, \frac{3}{2}\right)$ of $n^{\lambda}$. 
Proof. Taking the $L^{2}$ inner product of (3.12) with $D_{0}\left(n^{\lambda}\right) E_{\alpha}$, one gets, by integration by parts, that

$$
\begin{aligned}
\frac{d}{d t} \int_{\mathbb{T}^{3}} E_{\alpha}^{T} A_{0}\left(n^{\lambda}\right) E_{\alpha} d x= & \frac{2}{\lambda} \int_{\mathbb{T}^{3}} E_{\alpha}^{T} A_{0}\left(n^{\lambda}\right) G_{\alpha} d x+2 \int_{\mathbb{T}^{3}} E_{\alpha}^{T} A_{0}\left(n^{\lambda}\right) R_{\alpha}^{1} d x \\
& +2 \int_{\mathbb{T}^{3}} E_{\alpha}^{T} A_{0}\left(n^{\lambda}\right) R_{\alpha}^{2} d x \\
& +\int_{\mathbb{T}^{3}} E_{\alpha}^{T} \operatorname{div} A\left(W^{\lambda}\right) E_{\alpha} d x \\
= & \mathcal{I}_{\alpha}^{1}+\mathcal{I}_{\alpha}^{2}+\mathcal{I}_{\alpha}^{3}+\mathcal{I}_{\alpha}^{4}
\end{aligned}
$$

with $\operatorname{div} A\left(W^{\lambda}\right)=\partial_{t} A_{0}\left(n^{\lambda}\right)+\sum_{i=1}^{3} \partial_{x_{i}}\left(A_{0}\left(n^{\lambda}\right) A_{i}\left(W^{\lambda}\right)\right)$. Recalling that

$$
A_{0}\left(n^{\lambda}\right)=\left(\begin{array}{ccc}
h^{\prime}\left(n^{\lambda}\right) & 0 & 0 \\
0 & n^{\lambda} \mathbf{I}_{3 \times 3} & 0 \\
0 & 0 & n^{\lambda} \mathbf{I}_{3 \times 3}
\end{array}\right)
$$

and $n^{\lambda}=n_{\lambda}+N=1+N$, it is obvious that

$$
\begin{aligned}
\mathcal{I}_{\alpha}^{1} & =\frac{2}{\lambda} \int_{\mathbb{T}^{3}} n^{\lambda} E_{\alpha}^{T} G_{\alpha} d x \\
& =\frac{2}{\lambda} \int_{\mathbb{T}^{3}} n^{\lambda} U_{\alpha} \cdot \nabla \Phi_{\alpha} d x \\
& =\frac{2}{\lambda} \int_{\mathbb{T}^{3}}(1+N) U_{\alpha} \cdot \nabla \Phi_{\alpha} d x \\
& =-\frac{2}{\lambda} \int_{\mathbb{T}^{3}} \operatorname{div} U_{\alpha} \cdot \Phi_{\alpha} d x+\frac{2}{\lambda} \int_{\mathbb{T}^{3}} N U_{\alpha} \cdot \nabla \Phi_{\alpha} d x \\
& \leq-\frac{2}{\lambda} \int_{\mathbb{T}^{3}} \operatorname{div} U_{\alpha} \cdot \Phi_{\alpha} d x+\frac{2}{\lambda}\|N\|_{\infty} \int_{\mathbb{T}^{3}}\left|U_{\alpha} \| \nabla \Phi_{\alpha}\right| d x \\
& \leq-\frac{2}{\lambda} \int_{\mathbb{T}^{3}} \operatorname{div} U_{\alpha} \cdot \Phi_{\alpha} d x+\frac{2}{\lambda}\|N\|_{s}\left\|U_{\alpha}\right\|\left\|\nabla \Phi_{\alpha}\right\| .
\end{aligned}
$$

Recalling $n_{\lambda}=1$ and $N=n^{\lambda}-n_{\lambda}$, from (1.1) and (3.8), we have

$$
\operatorname{div} U=-\partial_{t} N-\operatorname{div}\left(u^{\lambda} N\right) .
$$

Then, from (3.11) we have

$$
\begin{aligned}
-\frac{2}{\lambda} \int_{\mathbb{T}^{3}} \operatorname{div} U_{\alpha} \cdot \Phi_{\alpha} d x= & \frac{2}{\lambda} \int_{\mathbb{T}^{3}} \partial_{t} N_{\alpha} \cdot \Phi_{\alpha} d x-\frac{2}{\lambda} \int_{\mathbb{T}^{3}}\left(u^{\lambda} N\right)_{\alpha} \cdot \nabla \Phi_{\alpha} d x \\
= & \frac{2}{\lambda} \int_{\mathbb{T}^{3}}\left(\lambda \partial_{t} \Delta \Phi_{\alpha}-\lambda^{2} \partial_{t} \Delta Q_{\alpha}\right) \cdot \Phi_{\alpha} d x-\frac{2}{\lambda} \int_{\mathbb{T}^{3}}\left(u^{\lambda} N\right)_{\alpha} \cdot \nabla \Phi_{\alpha} d x \\
= & -\frac{d}{d t} \int_{\mathbb{T}^{3}}\left|\nabla \Phi_{\alpha}\right|^{2} d x+2 \lambda \int_{\mathbb{T}^{3}} \partial_{t} \nabla Q_{\alpha} \cdot \nabla \Phi_{\alpha} d x \\
& -\frac{2}{\lambda} \int_{\mathbb{T}^{3}}(U N)_{\alpha} \cdot \nabla \Phi_{\alpha} d x-\frac{2}{\lambda} \int_{\mathbb{T}^{3}}\left(u_{\lambda} N\right)_{\alpha} \cdot \nabla \Phi_{\alpha} d x \\
\leq & -\frac{d}{d t} \int_{\mathbb{T}^{3}}\left|\nabla \Phi_{\alpha}\right|^{2} d x+2 \lambda\left\|\partial_{t} \nabla Q_{\alpha}\right\|\left\|\nabla \Phi_{\alpha}\right\|
\end{aligned}
$$


QUASI-NEUTRAL LIMIT OF EULER-POISSON SYSTEM COUPLED TO A MAGNETIC FIELD

$$
\begin{aligned}
& +\frac{C}{\lambda}\|U\|_{s}\|N\|_{s}\left\|\nabla \Phi_{\alpha}\right\|-\frac{2}{\lambda} \int_{\mathbb{T}^{3}}\left(u_{\lambda} N\right)_{\alpha} \cdot \nabla \Phi_{\alpha} d x \\
\leq & -\frac{d}{d t} \int_{\mathbb{T}^{3}}\left|\nabla \Phi_{\alpha}\right|^{2} d x+C\left\|\nabla \Phi_{\alpha}\right\|^{2}+\lambda^{2}+\frac{C}{\lambda}\|U\|_{s}\|N\|_{s}\left\|\nabla \Phi_{\alpha}\right\| \\
& -\frac{2}{\lambda} \int_{\mathbb{T}^{3}}\left(u_{\lambda} N\right)_{\alpha} \cdot \nabla \Phi_{\alpha} d x .
\end{aligned}
$$

Using (3.11) and $\operatorname{div} u_{\lambda}=0$, we have, by part by integrate, that

$$
\begin{aligned}
-\frac{2}{\lambda} \int_{\mathbb{T}^{3}}\left(u_{\lambda} N\right)_{\alpha} \cdot \nabla \Phi_{\alpha} d x= & -2 \int_{\mathbb{T}^{3}}\left(u_{\lambda} \Delta \Phi\right)_{\alpha} \cdot \nabla \Phi_{\alpha} d x-2 \lambda \int_{\mathbb{T}^{3}}\left(u_{\lambda} \Delta Q\right)_{\alpha} \cdot \nabla \Phi_{\alpha} d x \\
\leq & -2 \int_{\mathbb{T}^{3}}\left(u_{\lambda} \Delta \Phi\right)_{\alpha} \cdot \nabla \Phi_{\alpha} d x+C\left\|\nabla \Phi_{\alpha}\right\|^{2}+\lambda^{2} \\
= & -2 \int_{\mathbb{T}^{3}} u_{\lambda} \Delta \Phi_{\alpha} \cdot \nabla \Phi_{\alpha} d x+C\left\|\nabla \Phi_{\alpha}\right\|^{2}+\lambda^{2} \\
& -2 \int_{\mathbb{T}^{3}}\left[\left(u_{\lambda} \Delta \Phi\right)_{\alpha}-u_{\lambda} \Delta \Phi_{\alpha}\right] \cdot \nabla \Phi_{\alpha} d x \\
= & 2 \int_{\mathbb{T}^{3}} \nabla u_{\lambda}:\left(\nabla \Phi_{\alpha} \otimes \nabla \Phi_{\alpha}\right) d x+C\left\|\nabla \Phi_{\alpha}\right\|^{2}+\lambda^{2} \\
& -2 \int_{\mathbb{T}^{3}}\left[\left(u_{\lambda} \Delta \Phi\right)_{\alpha}-u_{\lambda} \Delta \Phi_{\alpha}\right] \cdot \nabla \Phi_{\alpha} d x \\
\leq & C\|\nabla\|_{s}^{2}+\lambda^{2} .
\end{aligned}
$$

where we have used the formulation

$$
\Delta \Phi_{\alpha} \cdot \nabla \Phi_{\alpha}=\operatorname{div}\left(\nabla \Phi_{\alpha} \otimes \nabla \Phi_{\alpha}\right)-\frac{1}{2} \nabla\left|\nabla \Phi_{\alpha}\right|^{2} .
$$

Then we can show that

$$
\begin{aligned}
\mathcal{I}_{\alpha}^{1} \leq & -\frac{d}{d t} \int_{\mathbb{T}^{3}}\left|\nabla \Phi_{\alpha}\right|^{2} d x+C\|\nabla \Phi\|_{s}^{2}+\frac{C}{\lambda}\|U\|_{s}\|N\|_{s}\left\|\nabla \Phi_{\alpha}\right\| \\
& +\frac{2}{\lambda}\|E\|_{s}\left\|U_{\alpha}\right\|\left\|\nabla \Phi_{\alpha}\right\|+\lambda^{2} .
\end{aligned}
$$

For $\mathcal{I}_{\alpha}^{2}$ and $\mathcal{I}_{\alpha}^{3}$, they are simply estimated as

$$
\begin{aligned}
& \mathcal{I}_{\alpha}^{2}=C \int_{\mathbb{T}^{3}}\left|E_{\alpha}\left\|R_{\alpha}^{1} \mid d x \leq C\right\| E_{\alpha}\|\| R_{\alpha}^{1} \|,\right. \\
& \mathcal{I}_{\alpha}^{3}=C \int_{\mathbb{T}^{3}}\left|E_{\alpha}\left\|R_{\alpha}^{1} \mid d x \leq C\right\| E_{\alpha}\|\| R_{\alpha}^{2} \| .\right.
\end{aligned}
$$

Moreover, we have

$$
\left|\operatorname{div} A\left(W^{\lambda}\right)\right| \leq C\left(1+\|E\|_{s}\right) .
$$

Then $\mathcal{I}_{\alpha}^{4}$ can be estimated as

$$
\mathcal{I}_{\alpha}^{4} \leq\left\|\operatorname{div} A\left(W^{\lambda}\right)\right\|_{\infty} \int_{\mathbb{T}^{3}} E_{\alpha}^{T} E_{\alpha} d x \leq C\left(1+\|E\|_{s}\right)\left\|E_{\alpha}\right\|^{2} .
$$

Now, substituting the inequalities (3.15)-(3.18) into (3.14) gives (3.13).

Set

$$
\mathcal{D}=\mathcal{D}(t)=\frac{\|E\|_{s}+\|\nabla \Phi\|_{s}}{\lambda} .
$$


Then, for the inequality in Lemma 3.1. we have the following claim.

Lemma 3.2. For any $\lambda \in(0,1)$, we have

$$
\frac{d}{d t} \int_{\mathbb{T}^{3}}\left(E_{\alpha}^{T} A_{0}\left(n^{\lambda}\right) E_{\alpha}+\left|\nabla \Phi_{\alpha}\right|^{2}\right) d x \leq C\left(1+\mathcal{D}^{s}\right)\left(\|E\|_{s}^{2}+\|\nabla \Phi\|_{s}^{2}\right)+\lambda^{2} .
$$

Proof. It is obviously that

$$
\begin{aligned}
& \frac{C}{\lambda}\|U\|_{s}\|N\|_{s}\left\|\nabla \Phi_{\alpha}\right\| \leq C \mathcal{D}\left(\|E\|_{s}^{2}+\|\nabla \Phi\|_{s}^{2}\right) \\
& \frac{2}{\lambda}\|E\|_{s}\left\|U_{\alpha}\right\|\left\|\nabla \Phi_{\alpha}\right\| \leq C \mathcal{D}\left(\|E\|_{s}^{2}+\|\nabla \Phi\|_{s}^{2}\right) \\
& \left(1+\|E\|_{s}\right)\left\|E_{\alpha}\right\|^{2} \leq C\left(1+\|\mathcal{D}\|_{s}^{s}\right)\|E\|_{s}^{2}
\end{aligned}
$$

Next we estimate $\left\|E_{\alpha}\right\|\left\|R_{\alpha}^{1}\right\|$. We use the boundedness of $\left\|\left(n_{\lambda}, u_{\lambda}, B_{\lambda}\right)\right\|_{s+1}=$ $\left\|\left(1, u^{0}, B^{0}\right)\right\|_{s+1}$ indicated in Proposition 2.3 to conclude that

$$
\begin{aligned}
\left\|R_{\alpha}^{1}\right\| \leq & C \sum_{i=1}^{3}\left\|u_{i}^{\lambda}-u_{\lambda i}\right\|_{|\alpha|}\left\|\partial_{x_{i}} W_{\lambda}\right\|_{s}+C \sum_{i=1}^{3}\left\|h^{\prime}\left(n^{\lambda}\right)-h^{\prime}\left(n_{\lambda}\right)\right\|\left\|_{|\alpha|}\right\| \partial_{x_{i}} W_{\lambda} \|_{s} \\
& +C \sum_{i=1}^{3}\left\|n^{\lambda}-n_{\lambda}\right\|_{|\alpha|}\left\|\partial_{x_{i}} W_{\lambda}\right\|_{s}+C \sum_{i=1}^{3}\left\|\frac{G_{i}^{\lambda}}{n^{\lambda}}-\frac{G_{\lambda i}}{n_{\lambda}}\right\|_{|\alpha|}\left\|\partial_{x_{i}} W_{\lambda}\right\|_{s} \\
\leq & C\|U\|_{|\alpha|}+C\left(1+\mathcal{D}^{s}\right)\|N\|_{|\alpha|}+C\|N\|_{|\alpha|}+C\left(1+\mathcal{D}^{s}\right)\left(\|N\|_{|\alpha|}+\|B\|_{|\alpha|}\right) \\
\leq & C\left(1+\mathcal{D}^{s}\right)\|E\|_{s} .
\end{aligned}
$$

In a similar spirit, $\left\|R_{\alpha}^{2}\right\|$ is estimated as

$$
\begin{aligned}
\left\|R_{\alpha}^{2}\right\| \leq & C \sum_{i=1}^{3}\left\|u_{i}^{\lambda} \partial_{x_{i}} E_{\alpha}-\left(u_{\lambda i} \partial_{x_{i}} E\right)_{\alpha}\right\| \\
& +C \sum_{i=1}^{3}\left\|h^{\prime}\left(n^{\lambda}\right) \partial_{x_{i}} N_{\alpha}-\left(h^{\prime}\left(n^{\lambda}\right) \partial_{x_{i}} N\right)_{\alpha}\right\| \\
& +C \sum_{i=1}^{3}\left\|n^{\lambda} \partial_{x_{i}} U_{\alpha}-\left(n^{\lambda} \partial_{x_{i}} U\right)_{\alpha}\right\| \\
& +C \sum_{i=1}^{3}\left\|\frac{\left(G_{i}^{\lambda}\right)^{T}}{n^{\lambda}} \partial_{x_{i}} H_{\alpha}-\left(\frac{\left(G_{i}^{\lambda}\right)^{T}}{n_{\lambda}} \partial_{x_{i}} H\right)_{\alpha}\right\| \\
& +C \sum_{i=1}^{3}\left\|\frac{G_{i}^{\lambda}}{n^{\lambda}} \partial_{x_{i}} U_{\alpha}-\left(\frac{G_{i}^{\lambda}}{n_{\lambda}} \partial_{x_{i}} U\right)_{\alpha}\right\| \\
\leq & C \sum_{i=1}^{3}\left\|u_{i}^{\lambda}\right\|_{s}\left\|\partial_{x_{i}} E\right\|_{|\alpha|-1}+C \sum_{i=1}^{3}\left\|h^{\prime}\left(n^{\lambda}\right)\right\|_{s}\left\|\partial_{x_{i}} N\right\|_{|\alpha|-1} \\
& +C \sum_{i=1}^{3}\left\|n^{\lambda}\right\|_{s}\left\|\partial_{x_{i}} U\right\|_{|\alpha|-1}+C \sum_{i=1}^{3}\left\|\frac{\left(G_{i}^{\lambda}\right)^{T}}{n^{\lambda}}\right\|_{s}\left\|\partial_{x_{I}} H\right\|_{|\alpha|-1} \\
& +C \sum_{i=1}^{3}\left\|\frac{G_{i}^{\lambda}}{n^{\lambda}}\right\|_{s}\left\|\partial_{x_{i}} U\right\|_{|\alpha|-1}
\end{aligned}
$$


QUASI-NEUTRAL LIMIT OF EULER-POISSON SYSTEM COUPLED TO A MAGNETIC FIELD

$$
\begin{aligned}
\leq & C \sum_{i=1}^{3}\left(\left\|u_{i}^{\lambda}-u_{\lambda i}\right\|_{s}+\left\|u_{\lambda i}\right\|_{s}\right)\|E\|_{|\alpha|}+C\left(1+\|N\|_{s}^{s}\right)\|N\|_{|\alpha|} \\
& +C\left(\left\|n^{\lambda}-n_{\lambda}\right\|_{s}+\left\|n_{\lambda}\right\|_{s}\right)\|U\|_{|\alpha|}+C\left(1+\|E\|_{s}^{s}\right)\|E\|_{|\alpha|} \\
\leq & C\left(1+\mathcal{D}^{s}\right)\|E\|_{s} .
\end{aligned}
$$

This completes the proof of Lemma 3.2

Note that $C^{-1}\left\|E_{\alpha}\right\|^{2} \leq \int_{\mathbb{T}^{3}} E_{\alpha}^{T} A_{0}\left(n^{\lambda}\right) E_{\alpha} d x \leq C\left\|E_{\alpha}\right\|^{2}$. We integrate (3.19) from 0 to $t$ with $[0, t] \subset\left[0, \min \left\{T_{\lambda}, T_{* *}\right\}\right]$ to obtain

$$
\left\|E_{\alpha}(t)\right\|^{2}+\left\|\nabla \Phi_{\alpha}(t)\right\|^{2} \leq C \int_{0}^{t}\left(1+\mathcal{D}^{s}\right)\left(\|E\|_{s}^{2}+\|\nabla \Phi\|_{s}^{2}\right) d \tau+C \lambda^{2} .
$$

Here we have used the fact that the initial data constructed in Theorem 2.4 Summing up the last inequality over all satisfying $|\alpha| \leq s$, we get

$$
\|E(t)\|_{s}^{2}+\|\nabla \Phi(t)\|_{s}^{2} \leq C \int_{0}^{t}\left(1+\mathcal{D}^{s}\right)\left(\|E\|_{s}^{2}+\|\nabla \Phi\|_{s}^{2}\right) d \tau+C T_{* *} \lambda^{2} .
$$

Applying Gronwall's lemma to (3.23), we obtain

$$
\|E(t)\|_{s}^{2}+\|\nabla \Phi(t)\|_{s}^{2} \leq C T_{* *} \lambda^{2} e^{C \int_{0}^{t}\left(1+\mathcal{D}^{s}\right) d \tau}
$$

In view of $\|E\|_{s}+\|\nabla \Phi\|_{s}=\lambda \mathcal{D}$, it follows from (3.24) that

$$
D^{2}(t) \leq C T_{* *} e^{C \int_{0}^{t}\left(1+\mathcal{D}^{s}\right) d \tau} \equiv \Gamma(t) .
$$

Thus, it holds that

$$
\Gamma^{\prime}(t)=C\left(1+\mathcal{D}^{s}\right) \Gamma(t) \leq C \Gamma(t)+C \Gamma^{\frac{s+2}{2}}(t) .
$$

Applying the nonlinear Gronwall-type inequality in 25] to the last inequality yields

$$
\Gamma(t) \leq e^{C T_{* *}}
$$

for $t \in\left[0, \min \left\{T_{\lambda}, T_{* *}\right\}\right]$ if we choose $T_{* *}>0$ (independent of $\lambda$ ) so small that

$$
\Gamma(0)=C T_{* *}<e^{-C T_{* *}} .
$$

Then, because of (3.25), there exists a positive constant $M$, independent of $\lambda$, such that

$$
\mathcal{D}(t) \leq M
$$

for $t \in\left[0,\left\{T_{\lambda}, T_{* *}\right\}\right]$. Finally, from (3.24), (3.26) and the definition of $(E, \nabla \Phi)$, we conclude the proof of Theorem 2.4 ,

\section{Acknowledgments :}

J. Yang's research was partially supported by the Joint Funds of the National Natural Science Foundation of China (Grant No. U1204103). 


\section{REFERENCES}

[1] Y. Brenier, Convergence of the Vlasov-Poisson system to the incompressible Euler equations, Comm. Partial Differential Equations, 25 (2000), 737-754. 2

[2] S. Cordier and E. Grenier, Quasineutral limit of an Euler-Poisson system arising from plasma physics, Comm. Partial Differential Equations, 25(2000), 1099-1113. 2

[3] J. P. Cox, R. T. Giuli, Principles of stellar structure, I.,II., Gordon and Breach, New York, 1968. 1

[4] J. I. Díaz and M. B. Lerena, On the inviscid and non-resistive limit for the equations of incompressible magnetohydrodynamics, Math. Models Meth. Appl. Sci. 12 (2002), 14011419. 5

[5] D. Donatelli, P. Marcati, Analysis of oscillations and defect measures for the quasineutral limit in plasma physics, Arch. Ration. Mech. Anal., 206 (2012), 159-188. 2

[6] P. Federbush, T. Luo, J. Smoller, Existence of Magnetic Compressible Fluid Stars, Arch. Rational Mech. Anal. 215 (2015), 611-631. 1

[7] I. Gasser, C. D. Levermore, P. Markowich, C. Schmeiser, The initial time layer problem and the quasineutral limit in the semiconductor drift-diffusion model, Europ. J. Appl. Math. 12 (2001), 497-512. 2

[8] I. Gasser, L. Hsiao, P. Markowich, S. Wang, Quasineutral limit of a nonlinear drift-diffusion model for semiconductor models, J. Math. Anal. Appl. 268 (2002), 184-199. 2

[9] A. Jüngel, Y. J. Peng, A hierarchy of hydrodynamic models for plasmas: Quasi-neutral limits in the drift-diffusion equations. Asympt. Anal. 28 (2001), 49-73. 2

[10] Q. C. Ju, F. C. Li, H. L. Li, The quasineutral limit of compressible Navier-Stokes-Poisson system with heat conductivity and general initial data, J. Differential Equations, 247 (2009), 203-224. 2

[11] E. Grenier, Defect measures of the Vlasov-Poisson system, Comm. Partial Differential Equations 20(1995), 1189-1215. 2

[12] E. Grenier, Oscillations in quasineutral plasmas. Comm. Partial Differential Equations, 21 (1996), 363-394. 2

[13] E. Grenier, Pseudodifferential estimates of singular perturbations, Commun. Pure Appl. Math. 50 (1997), 821-865. 2

[14] H. L. Li, C. K. Lin, Zero Debye length asymptotic of the quantum hydrodynamic model for semiconductors, Comm. Math. Phys. 256 (2005), 195-212. 2

[15] Y. P. Li, Convergence of the nonisentropic Euler-Poisson equations to incompressible type Euler equations. J. Math. Anal. Appl.342 (2008), 1107-1125. 2

[16] Y. Li, Q. C. Ju, W. Q. Xu, Quasi-neutral limit of the full Navier-Stokes-Fourier-Poisson system, J. Differential Equations, 258 (2015), 3661-3687 2

[17] G. Loeper, Quasi-neutral limit of the Euler-Poisson and Euler-Monge-Ampère systems, Comm. Partial Differential Equations, 30 (2005), 1141-1167. 2

[18] A. Majda, Compressible Fluid Flow and Systems of Conservation Laws in Several Space Variables, Springer-Verlag, New-York, 1984. 3 4

[19] N. Masmoudi, From Vlasov-Poisson system to the incompressible Euler system, Comm. Partial Differential Equations, 26 (2001), 1913-1928. 2

[20] Y. J. Peng, Y. G. Wang, W. A. Yong, Quasi-neutral limit of the non-isentropic Euler-Poisson systems, Proc. Roy. Soc. Edinburgh, A136 (2006), 1013-1026. 2

[21] S. H. Shapiro, S. A. Teukolsky, Black Holes, White Dwarfs, and Neutron Stars, WILEYVCH, 2004. 1

[22] E. Stein, Singular Integrals and Differentiability, Princeton, Princeton Univ. Press, New Jersey, 1970. 圆

[23] S. Wang, Quasineutral limit of Euler-Poisson system with and without viscosity, Comm. Partial Differential Equations, 29 (2004), 419-456. 2

[24] S. Wang, S. Jiang, The convergence of the Navier-Stokes-Poisson system to the incompressible Euler equations, Comm. Partial Differential Equations, 31 (2006), 571-591. 2 
QUASI-NEUTRAL LIMIT OF EULER-POISSON SYSTEM COUPLED TO A MAGNETIC FIEL\#3

[25] W. A. Yong, Singular perturbations of first-order hyperbolic systems with stiff source terms, J. Differential Equations, 155 (1999), 89-132.2 611

[26] W. A. Yong, Basic aspects of hyperbolic relaxation systems, in Advances in the Theory of Shock Waves, H. Freistühler and A. Szepessy, eds., Progr. Nonlinear Differential Equations Appl. 47, Birkhäuser Boston, Boston, 2001, 259-305. 2 5 6

College of Mathematics and Information Science, North China University of Water Resources and Electric Power, Zhengzhou 450045, Henan Province, P. R. China

E-mail address: yangjianwei@ncwu.edu.cn 